\title{
Avanços e iniciativas na comunicação pela lente web 2.0 para a Lei de Acesso à Informação no Brasil
}

Simone Maria da Silva Lima - simonemslima@gmail.com ${ }^{1}$ Jairo Simião Dornelas - jairo@ufpe.br ${ }^{2}$

Resumo - Este artigo objetiva analisar a disponibilização de ferramentas de comunicação e interatividade, a partir da aplicação da Lei Federal 12.527 de 2011, conhecida como a lei de acesso à informação (LAI) no Brasil. A utilização da Internet passou a ser um canal obrigatório de comunicação e às instituições públicas precisam realizar ações de adequação à lei, bem como a integração com contexto ainda crescente da web 2.0. De acordo com os resultados deste estudo, a opção de compartilhamento das informações nas redes sociais ainda é pouco disponibilizada. O uso do chat para tirar dúvidas em tempo real não foi verificado em nenhum dos portais pesquisados, no entanto a maioria dos portais disponibiliza um formulário de fale conosco ou um email para contato. Os cidadãos passam a ter novas opções de acesso, no entanto, à questão da interatividade ainda tem muitas oportunidades de melhorias.

Palavras-chave: Lei de Acesso à Informação. Web 2.0. Comunicação e Interatividade.

\section{Web 2.0 lens to observes the Access to Information Law in Brazil in communication process: initiatives and advances}

\begin{abstract}
This article aims to analyze the availability of communication and interactivity tools, the from the application of Federal Law 12,527 of 2011, known as the access to information law (LAI) in Brazil. Internet use has become a mandatory communication channel and public institutions need to carry out adaptation to the law, as well as integration with the still growing context of web 2.0. According to results of this study, the option of sharing information on social networks is still poorly available and the use of chat to answer questions in real time was not verified in any of the portals surveyed; however, most portals provide a contact form or an email for contact. The citizens have new access options, however, the issue of interactivity still has many opportunities for improvements.
\end{abstract}

Keywords: Access to Information Law. Web 2.0. Communication and Interactivity. 


\section{Introdução}

A Lei I2.527 de Acesso à Informação (BRASIL, 20II), que entrou em vigor dia I6 de maio de 20I2, estabelece novas diretrizes para informações aos cidadãos. Para cumprimento da lei, os órgãos e entidades públicas deverão utilizar todos os meios de comunicação e instrumentos de que dispuserem, sendo obrigatória a divulgação na internet. Além disso, informações adicionais podem ser solicitadas pelos cidadãos e tem prazos pré-definidos para serem fornecidas. Assim a regra passa a ser a disponibilização das informações e o sigilo é agora a exceção.

A seu turno, na atualidade, a utilização da Internet passou a ser um canal obrigatório de comunicação e às instituições públicas precisam realizar ações de adequação à lei, bem como a integração com contexto ainda crescente da web 2.o. Não à toa este é um tema que desperta o interesse para estudo.

Nota-se que a web alcançara um crescimento considerável, com usuários estimados em muitos milhões, em práticas através das redes sociais, como Facebook ${ }^{\circledR}$ e LinkedIn ${ }^{\circledR}$; nos blogs e microblogs, como o Twitter ${ }^{\circledR}$; no compartilhamento de conteúdo do usuário, como o YouTube ${ }^{\circledR}$ e o Flickr ${ }^{\circledR}$. Já a web 2.0 fornece recursos sem precedentes para a construção das redes virtuais, onde o indivíduo pode colaborar coletivamente e comunicar-se globalmente (ORLIKOWSKI e WOERNER, 2009). Nessa tecnologia, são termos comuns: a colaboração, a utilização de um ambiente compartilhado, a descentralização, a comunicação e a interatividade (BANNON e SCHMIDT, I99I; O'REILLY, 2005, TAPIADOR et al, 2006; ORLIKOWSKI e WOERNER, 2009; McMILLAN, 2005).

Combinando estas duas vertentes e a partir da lei de acesso à informação, tem-se o surgimento de uma nova conjuntura que interliga a tecnologia da informação (TI) e os processos administrativos nas organizações públicas, com a abertura de novas possibilidades para comunicação.

É justo nesta perspectiva que este artigo objetiva analisar a aplicação da Lei Federal I2.527 de 20II, conhecida como a lei de acesso à informação (LAI) no Brasil, a partir da investigação de disponibilização de ferramentas de comunicação e interatividade associadas à web 2.0 para acomodação de informações nos portais de transparência nos estados e no Distrito Federal que já estão com a agenda da LAI supostamente regularizada.

\section{Revisão da Literatura}

Esta seção é iniciada com apresentação de conceitos relacionados a web 2.0, seguido pela temática da interatividade e comunicação. Ao final serão apresentadas as principais informações relacionadas à lei de acesso à informação e as novas diretrizes para disponibilizar dados aos cidadãos através da internet.

\subsection{Web 2.0}

Conforme O'Reilly (2005), o conceito de web 2.0 começou com uma sessão de brainstorming entre O'Reilly e a Internacional MediaLive. Mesmo sendo ainda um conceito em de- 
senvolvimento, pontos que podem ser discutidos, referem-se à evolução da Internet comparativamente com o modelo anterior. Dentre os principais termos utilizados sobre a web 2.0 estão: a colaboração, a utilização de um ambiente compartilhado, a descentralização, a comunicação e a interatividade (BANNON E SCHMIDT, I99I; O'REILLY, 2005, TAPIADOR et al, 2006; ORLIKOWSKI E WOERNER, 2009; McMILLAN, 2005).

Em geral, podem ser citados alguns itens que demonstram uma mudança na estrutura da Internet, quando comparadas as duas gerações da web, conforme mostra o Quadro I.

QUADRO I: Funcionalidades e aplicações web versus web 2.0

\begin{tabular}{|c|c|}
\hline Web & Web 2.0 \\
\hline Ofoto & Flickr \\
\hline Visualizações de páginas & \multirow{2}{*}{ Napster $^{\circledR}$} \\
\hline mp3.com & \\
\hline Sistemas de gerenciamento de conteúdo & Blogs \\
\hline Sites Pessoais & \\
\hline Publicação & \\
\hline
\end{tabular}

Fonte: Baseado em O’Reilly (2005).

As competências essenciais das organizações para web 2.0 incluem itens como: controles difíceis de recriar e que fiquem melhores quanto mais pessoas usarem; utilização dos usuários como co-desenvolvedores e aproveitamento da inteligência coletiva; além de interfaces leves com o usuário no desenvolvimento dos sistemas (O’REILLY, 2005).

Para Tapiador et al. (2006), o salto qualitativo para web 2.0 é a habilidade dos usuários fácil e remotamente poderem alterar conteúdos, bem como a feature da bidirecionalidade dos canais de comunicação, que ampliaram o potencial para participar de qualquer coisa na web 2.o.

O trabalho colaborativo suportado pela Internet (Internet Supported Collaborative Work - ISCW) é um novo tema que se faz presente na vida das pessoas, pois os usuários têm a possibilidade de tornarem-se componentes do trabalho global nessa web colaborativa. Algumas ferramentas, que podem ser citadas como parte das possibilidades de utilização em ambientes colaborativos são: bate-papos (chat's); correio eletrônico (e-mail); listas de discussão; fóruns; mensagens instantâneas; audio-conferência; video-conferência; compartilhamento de documentos e aplicativos; entre outros (BRITO, 2004).

\subsection{Comunicação e interatividade}

A comunicação e os processos envolvidos no fluxo de informações pessoais e organizacionais têm se desenvolvido em estudos e publicações diversas, e alguns elementos tomados como essenciais estão descritos nas diversas definições: emissor, codificação, mensagem, decodificação, receptor e feedback (STONER E FREEMAN, I985; ROBBINS, 1999).

De forma geral a comunicação envolve pessoas, significados compartilhados e lingua- 
gem simbólica em um processo de troca de informações (STONER E FREEMAN, 1985) e para que um sistema de comunicação organizacional se mostre eficiente, está envolvido que o processamento de informações possa levar em consideração as diferentes atividades, observando que atividades mais complexas, com maior nível de incerteza e interdependência exigem diferentes e apropriados requisitos nesse processamento das informações (HAMPTON, I992).

Para Rafaeli e Sudweeks (1997) a interatividade é uma qualidade variável de configurações da comunicação que expressa o grau com que a comunicação transcende a reação. Assim o conceito de interatividade dirige o foco para a intersecção do psicológico com o sociológico, mostra-se como ponte entre a comunicação de massa e a comunicação interpessoal, sendo à reunião da comunicação mediada e da comunicação direta, e o paradoxo da comunicação escrita versus falada.

Um grande número de novas aplicações e serviços com rica troca de informações multimídia e que mostram a evolução de um conhecimento agregado, passam cada vez mais a dominar a web e como exemplos dessas aplicações, temos os blogs, wikis, comunidades de código aberto, compartilhamento de fotos e vídeos e redes de negócios online e itens que facilitam a ação coletiva e a interação social online (Parameswaran e Whinston, 2007).

McMillan (2005) define interatividade baseando-se nas características do ambiente de comunicação que o tornam interativo, nos processos com as atividades de interação, e nas percepções que mostram como os usuários percebem ou não o ambiente de comunicação como sendo interativo. Ela identifica três diferentes "tipos" de interatividade: humano-a-humano, humano-computador e humano-conteúdo. Tal tipologia é exemplificada no Quadro 2 que versa sobre modos de visualização de diferentes formas de interatividade.

QUADRO 2: Diferentes formas de interatividade e seus exemplos

\begin{tabular}{|l|c|}
\hline \multicolumn{1}{|c|}{ Tipo de Interação } & Exemplos de Aplicações \\
\hline Humano-Humano & Participar de chat's (bate-papo); Enviar/receber $e$-mails \\
\hline Humano-Computador & Navegar em sites da internet; Utilizar ferramentas de busca \\
\hline Humano-Conteúdo & $\begin{array}{c}\text { Criar uma home page personalizada } \\
\text { Procurar notícias em diversos formatos de mídia }\end{array}$ \\
\hline
\end{tabular}

Fonte: Baseada em McMillan (2005).

Conforme indicado por Rafaeli e Sudweeks (1997) a interatividade no contexto da Comunicação Mediada por Computador (CMC) mostra-se como uma experiência de integração social e participação democrática, complementando que a interatividade é possível, mas nem sempre exercido na CMC.

\subsection{Lei de acesso à informação}

A lei de acesso à informação (LAI) é a lei I2.527 de I8 de novembro de 201 I que dispõe sobre os procedimentos a serem observados pela União, Estados, Distrito Federal e Municípios, com o fim de garantir o acesso a informações. Seu prazo de implementação foi de 6 meses entrando em vigor efetivamente no dia I6 de maio de $20 \mathrm{I} 2$ (CGU, 20II). 
Como princípios básicos da LAI (BRASIL, 20II), são elencados os seguintes pontos: I - observância da publicidade como preceito geral e do sigilo como exceção; II - divulgação de informações de interesse público, independentemente de solicitações; III - utilização de meios de comunicação viabilizados pela tecnologia da informação; IV - fomento ao desenvolvimento da cultura de transparência na administração pública; $\mathrm{V}$ - desenvolvimento do controle social da administração pública. Para cumprimento da lei os órgãos e entidades públicas deverão utilizar todos os meios e instrumentos legítimos de que dispuserem, sendo obrigatória a divulgação na internet. As informações adicionais solicitadas pelos cidadãos devem ser repassadas de acordo com os prazos legais.

A questão do acesso à informação e transparência já era tratado no país de outras formas: no artigo 5 da Constituição de I988; com a Lei de Responsabilidade Fiscal; Lei do Processo Administrativo; a Lei do Habeas Data e a Lei de Arquivos e a disponibilização de informações desde 2004 pelo Portal da Transparência do Governo Federal (CGU, 20II). A lei de acesso à Informação vem complementar e dispor mais detalhes sobre esse tema.

QUADRO 3: Cultura de Segredo versus Cultura de Acesso

\begin{tabular}{|c|c|}
\hline Cultura de Segredo & Cultura de Acesso \\
\hline $\begin{array}{l}\text { O cidadão só pode solicitar informações que the } \\
\text { digam respeito direto. }\end{array}$ & $\begin{array}{l}\text { O cidadão pode solicitar a informação pública sem } \\
\text { necessidade de justificativa. }\end{array}$ \\
\hline $\begin{array}{l}\text { Os dados podem ser utilizados indevidamente por } \\
\text { grupos de interesse. } \\
\text { Os cidadãos não estão preparados para exercer o } \\
\text { direito de acesso à informação. }\end{array}$ & A demanda do cidadão é vista como legítima. \\
\hline $\begin{array}{l}\text { A demanda do cidadão é um problema: sobrecarrega } \\
\text { os servidores e compromete outras atividades. }\end{array}$ & $\begin{array}{l}\text { São criados canais eficientes de comunicação entre } \\
\text { governo e sociedade. } \\
\text { Os servidores são permanentemente capacitados } \\
\text { para atuarem na implementação da política de aces- } \\
\text { so à informação. }\end{array}$ \\
\hline $\begin{array}{l}\text { Cabe sempre à chefia decidir pela liberação ou não da } \\
\text { informação. }\end{array}$ & $\begin{array}{l}\text { São estabelecidas regras claras e procedimentos } \\
\text { para a gestão das informações. }\end{array}$ \\
\hline $\begin{array}{l}\text { A informação é retida e até perdida. A gestão pública } \\
\text { perde em eficiência, o cidadão não exerce um direito } \\
\text { e o Estado não cumpre seu dever. }\end{array}$ & $\begin{array}{l}\text { O fluxo de informações favorece a tomada de deci- } \\
\text { sões, a boa gestão de políticas públicas e a inclusão } \\
\text { do cidadão. }\end{array}$ \\
\hline
\end{tabular}

Fonte: Autores - baseado em CGU (20II)

Conforme mostra o Quadro 3, há uma mudança envolvida trilhando da cultura do segredo para uma cultura do acesso, pois agora a regra é a disponibilização das informações e o sigilo é que passa a ser a exceção. Vale ressaltar que de forma geral, a informação pública somente pode ser classificada como sigilosa quando considerada imprescindível à segurança da sociedade (à vida, segurança ou saúde da população) ou do Estado (soberania nacional, relações internacionais, atividades de inteligência). 


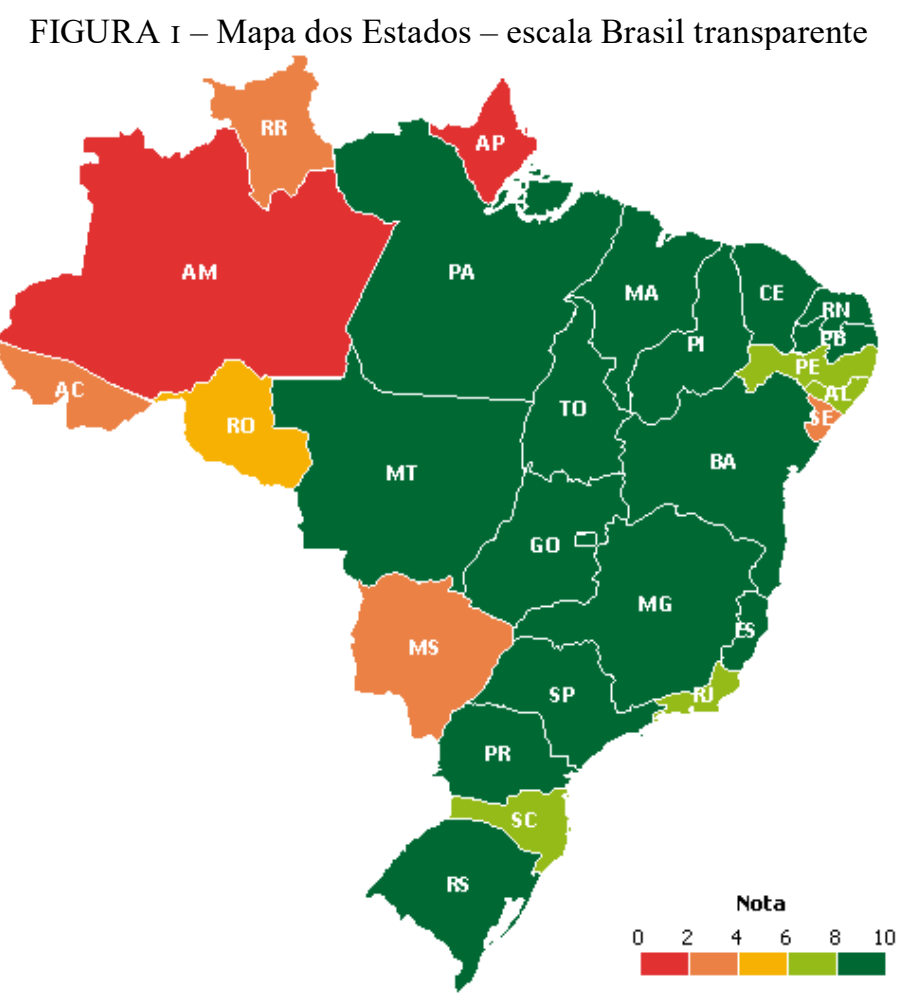

Fonte: BRASIL (2016).

A Figura I mostra um mapa que representa a Escala Brasil Transparente (EBT) a partir das notas dos estados e do Distrito Federal. A EBT que tem como objetivo medir a transparência pública em Estados e Municípios brasileiros e avaliar o grau de cumprimento de dispositivos da lei de acesso à informação. Através do Programa Brasil Transparente (BRASIL, 20I6), até fevereiro de 20I6, houveram I.576 adesões, com todos os estados e o Distrito Federal regulamentados.

QUADRO 4: Escala Brasil Transparente - Governos Estaduais

\begin{tabular}{|c|l|l|c|}
\hline Posição & \multicolumn{1}{|c|}{ Estado } & Nota \\
\hline $1-8$ & $\begin{array}{l}\text { Bahia } \\
\text { Distrito Federal } \\
\text { Espírito Santo } \\
\text { Goiás }\end{array}$ & $\begin{array}{l}\text { Maranhão } \\
\text { Minas Gerais } \\
\text { São Paulo } \\
\text { Tocantins }\end{array}$ & 10,0 \\
\hline 9 & Paraná & 9,31 \\
\hline 10 & Pará & 9,03 \\
\hline 11 & Rio Grande do Sul & 8,89 \\
\hline 12 & Paraíba & 8,75 \\
\hline 13 & Mato Grosso & 8,61 \\
\hline 14 & Piauí & 8,47 \\
\hline 15 & Rio Grande do Norte & 8,19 \\
\hline 16 & Ceará & 8,06 \\
\hline 17 & Alagoas & 7,92 \\
\hline 18 & Rio de Janeiro & 7,08 \\
\hline 19 & Santa Catarina & 6,94 \\
\hline
\end{tabular}




\begin{tabular}{|c|c|c|c|}
\hline 20 & \multicolumn{2}{|l|}{ Pernambuco } & 6,67 \\
\hline 21 & \multicolumn{2}{|l|}{ Rondônia } & 4,44 \\
\hline 22 & \multicolumn{2}{|l|}{ Acre } & 3,33 \\
\hline $23-24$ & Mato Grosso do Sul & Roraima & 2,5 \\
\hline 25 & \multicolumn{2}{|l|}{ Sergipe } & 2,08 \\
\hline 26 & \multicolumn{2}{|l|}{ Amazonas } & 1,39 \\
\hline 27 & \multicolumn{2}{|l|}{ Amapá } & 0 \\
\hline
\end{tabular}

Fonte: Baseado em BRASIL (20I6).

Observando o ranking dos Estados, conforme apresentado no Quadro 4, 7 Estados e o Distrito Federal apresentam nota máxima (IO,o). A metodologia das notas é baseada em I2 quesitos onde $25 \%$ da nota refere-se a regulamentação da lei de acesso à informação e $75 \%$ a efetiva existência e atuação dos sistemas de informação ao cidadão (SIC).

\section{Metodologia}

Essa pesquisa é qualificada como uma pesquisa documental, de viés descritivo, operacionalizada com dados primários e suplementada por uma etapa de observação não obtrusiva, baseada em uma grade de análise interpretativa, tendo, portanto, uma abordagem qualitativa (GIL, 2009; MARCONI E LAKATOS, 2007). Através da observação e registro das informações, buscou-se mostrar a real disponibilização de ferramentas de comunicação e interatividade em portais governamentais, sondando a facilitação de acesso a partir de aplicações que graçam na web 2.o. O inventário analítico-interpretativo arquitetado para compilar e sumarizar os resultados teve inspiração nas codificações peculiares a grounded theory (STRAUSS E CORBIN, 2008).

A tarefa de coleta de dados foi realizada a partir de dados disponíveis nos portais da transparência dos 26 estados brasileiros e o Distrito Federal que compõem a população deste estudo.

Baseando-se em Creswell (2007), adotou-se como procedimento de registro de dados um protocolo observacional (grade mais ação de codificação), identificando previamente os dados a serem registrados e os procedimentos para o registro desses dados. Como vantagem destaca-se que esse tipo de fonte de informação pode ser acessado de acordo com a conveniência do tempo disponível do pesquisador, bem como o fato de que evidências escritas poupam tempo e gastos do pesquisador. No Quadro 5, estão identificados os endereços eletrônicos dos portais avaliados. 
QUADRO 5: Portais de Transparência 20I6

\begin{tabular}{|l|l|l|}
\hline & Estado & Portal da transparência \\
\hline 1 & Bahia & www.transparencia.ba.gov.br/ \\
\hline 2 & Distrito Federal & www.transparencia.df.gov.br/ \\
\hline 3 & Espírito Santo & www.transparencia.es.gov.br/ \\
\hline 4 & Goiás & www.transparencia.goias.gov.br/ \\
\hline 5 & Maranhão & www.transparencia.ma.gov.br/ \\
\hline 6 & Minas Gerais & www.transparencia.mg.gov.br/ \\
\hline 7 & São Paulo & www.transparencia.sp.gov.br/ \\
\hline 8 & Tocantins & www.transparencia.to.gov.br/ \\
\hline 9 & Paraná & www.portaldatransparencia.pr.gov.br/ \\
\hline 10 & Pará & www.transparencia.pa.gov.br/ \\
\hline 11 & Rio Grande do Sul & www.transparencia.rs.gov.br/ \\
\hline 12 & Paraíba & www.transparencia.pb.gov.br/ \\
\hline 13 & Mato Grosso & www.transparencia.mt.gov.br/ \\
\hline 14 & Piauí & www.transparencia.pi.gov.br/ \\
\hline 15 & Rio Grande do Norte & www.transparencia.rn.gov.br/ \\
\hline 16 & Ceará & www.transparencia.ce.gov.br/ \\
\hline 17 & Alagoas & www.transparencia.al.gov.br/ \\
\hline 18 & Rio de Janeiro & www.transparencia.rj.gov.br/ \\
\hline 19 & Santa Catarina & www.sef.sc.gov.br/transparencia \\
\hline 20 & Pernambuco & www.transparencia.pe.gov.br/ \\
\hline 21 & Rondônia & www.transparencia.ro.gov.br/ \\
\hline 22 & Acre & www.sefaznet.ac.gov.br/transparencia/ \\
\hline 23 & Mato Grosso do Sul & www.portaldatransparencia.ms.gov.br/ $/$ \\
\hline 24 & Roraima & www.portal.rr.gov.br/ \\
\hline 25 & Sergipe & www.transparenciasergipe.se.gov.br/ \\
\hline 26 & Amazonas & www.transparencia.am.gov.br/ \\
\hline 27 & Amapá & www.transparencia.ap.gov.br \\
\hline & & Os Autores (2or6). \\
\hline
\end{tabular}

Fonte: Os Autores (20I6).

\section{Análise de dados}

Avaliando os pontos referentes à divulgação de informações de interesse público, independentemente de solicitações e a utilização de meios de comunicação na web 2.0 viabilizados pela tecnologia da informação, foram verificados os portais da transparência de cada estado e cinco categorias foram detalhadas: dados para consulta livre; email/fale conosco exclusivo; fale conosco/ ouvidoria geral; chat: redes sociais. O Quadro 6 mostra um resumo das informações coletadas. A ordem de descrição dos estados segue a classificação decrescente das notas recebidas no ranking de estados, mostrados anteriormente no Quadro 4. 
QUADRO 6: Comunicação e interatividade nos portais de transparência

\begin{tabular}{|c|c|c|c|c|c|c|}
\hline & Estado & $\begin{array}{c}\text { Dados para } \\
\text { consulta livre }\end{array}$ & $\begin{array}{c}\text { Email/ } \\
\text { Fale Conosco } \\
\text { Exclusivo } \\
\end{array}$ & $\begin{array}{c}\text { Email/Fale } \\
\text { conosco } \\
\text { Geral } \\
\end{array}$ & Chat & $\begin{array}{c}\text { Compartilham. } \\
\text { em Redes So- } \\
\text { ciais }\end{array}$ \\
\hline 1 & Bahia & SIM & SIM & - & $\mathrm{NÃO}$ & NÃO \\
\hline 2 & Distrito Federal & SIM & SIM & - & $\mathrm{NÃO}$ & $\mathrm{NÃO}$ \\
\hline 3 & Espírito Santo & SIM & SIM & - & $\mathrm{NÃO}$ & NÃO \\
\hline 4 & Goiás & SIM & SIM & - & NÃO & $\mathrm{NÃO}$ \\
\hline 5 & Maranhão & SIM & SIM & - & $\mathrm{NA \tilde {O }}$ & $\mathrm{NÃO}$ \\
\hline 6 & Minas Gerais & SIM & $\overline{\text { SIM }}$ & - & $\mathrm{NÃO}$ & SIM \\
\hline 7 & São Paulo & SIM & SIM & - & NÃO & NÃO \\
\hline 8 & Tocantins & SIM & $\overline{\text { SIM }}$ & - & NÃO & NÃO \\
\hline 9 & Paraná & SIM & $\mathrm{NÃO}$ & SIM & NÃO & NÃO \\
\hline 10 & Pará & SIM & $\mathrm{NÃO}$ & SIM & NÃO & $\mathrm{NÃO}$ \\
\hline 11 & Rio Grande do Sul & SIM & SIM & - & $\mathrm{NÃO}$ & NÃO \\
\hline 12 & Paraíba & SIM & SIM & - & NÃO & SIM \\
\hline 13 & Mato Grosso & SIM & SIM & - & $\mathrm{NÃO}$ & $\mathrm{NÃO}$ \\
\hline 14 & Piauí & SIM & SIM & - & $\mathrm{NÃO}$ & $\overline{\mathrm{NÃO}}$ \\
\hline 15 & Rio Grande do Norte & SIM & SIM & - & $\mathrm{NÃO}$ & $\mathrm{NÃO}$ \\
\hline 16 & Ceará & SIM & SIM & - & $\mathrm{NA \tilde {O }}$ & $\mathrm{NÃO}$ \\
\hline 17 & Alagoas & SIM & SIM & - & $\mathrm{NÃO}$ & SIM \\
\hline 18 & Rio de Janeiro & SIM & SIM & - & NÃO & NÃO \\
\hline 19 & Santa Catarina & SIM & SIM & - & $\mathrm{NÃO}$ & NÃO \\
\hline 20 & Pernambuco & SIM & SIM & - & $\mathrm{NÃO}$ & $\mathrm{NÃO}$ \\
\hline 21 & Rondônia & SIM & SIM & - & $\mathrm{NÃO}$ & $\mathrm{NÃO}$ \\
\hline 22 & Acre & SIM & $\overline{\mathrm{NÂO}}$ & $\overline{\mathrm{NÃO}}$ & NÃO & NÃO \\
\hline 23 & Mato Grosso do Sul & SIM & $\mathrm{NÃO}$ & SIM & NÃO & $\mathrm{NÃO}$ \\
\hline 24 & Roraima & SIM & SIM & - & $\mathrm{NA \tilde {O }}$ & $\mathrm{NÃO}$ \\
\hline 25 & Sergipe & $\overline{\text { SIM }}$ & $\mathrm{NÂO}$ & SIM & $\mathrm{NÃO}$ & $\mathrm{NÃO}$ \\
\hline 26 & Amazonas & SIM & SIM & - & $\mathrm{NÃO}$ & $\mathrm{NÃO}$ \\
\hline 27 & Amapá & $\overline{\text { SIM }}$ & SIM & - & $\mathrm{NA \tilde {O }}$ & $\mathrm{NÃO}$ \\
\hline
\end{tabular}

a) Dados para consulta livre: Foi avaliada a disponibilidade de dados para consulta geral e de livre acesso, sem necessidade de nenhum tipo de cadastro prévio. Em todos os portais de transparência verificados são disponibilizadas informações sobre as ações do estado, no entanto, o nível de detalhamento, varia bastante em cada portal. Nas informações sobre despesas com pessoal, por exemplo, alguns portais mostram detalhamentos nominais, outros portais as divisões por função. Referente a valores de receitas e despesas e outras informações acontece o mesmo: não foi constatado nenhum tipo de padrão ou normatização em relação à quais temas são prioritárias para divulgação de livre consulta nos portais avaliados.

Muitas das informações que já eram publicadas antes da LAI e que são de conhecimento público, como o diário oficial, também podem ser encontradas nos portais da transparência. Quando os dados disponíveis estão em formato pdf ou a visualização não permite a opção de copiar ou baixar em formatos como o Microsoft Word/Excel, não há uma facilidade para usá-las como fonte para outras aplicações.

b) Email/Fale Conosco Exclusivo: Foi verificada a existência de uma opção de comunicação com os estados através de envio de email diretamente pelo portal da transparência ou disponibilização de email exclusivamente para contato dentro do portal da transparência. Apenas os estados do Paraná, Pará, Acre, Mato Grosso do Sul e Sergipe não disponibilizam esta opção direta que poderia ser utilizada para pedido de informações adicionais, dúvidas e outras necessidades dos usuários. 
c) Fale conosco/ Ouvidoria Geral: Para os estados que não disponibilizam diretamente no portal da transparência a opção de enviar um email ou deixar uma mensagem através de uma ferramenta como o "fale conosco", observou-se que em alguns casos o portal da transparência tem a opção de direcionar o usuário para um contato através de email geral do estado ou ouvidoria. Nestes casos, o email não é exclusivo para informações do Portal da Transparência, mas a comunicação pode ser efetivada através desse canal. No entanto, só foram considerados os casos em que o email ou fale conosco mesmo que não seja exclusivo, tenha um link de direcionamento no portal da transparência do estado. Apenas no portal de transparência do Acre não há nenhum tipo de opção de comunicação para dúvidas/informações.

Ainda de acordo com a LAI, informações adicionais podem ser solicitadas pelo cidadão. Nesse caso pode ser utilizado o e-SIC, Sistema Eletrônico do Serviço de Informações ao Cidadão, que é um sistema que permite solicitar informações não disponíveis nos portais.

d) Chat: Foi pesquisado se algum portal de transparência dos estados avaliados tinha a ferramenta de chat online, para contato direto do cidadão e o governo. Constatou-se que nenhum dos estados oferece essa opção de comunicação. Alguns governos podem ter a opção de chat, mas não foi verificado link diretamente nos sites dos portais de transparência.

e) Redes Sociais (Facebook ${ }^{\circledR} /$ Google $^{\circledR} /{ }^{\circledR}$ Instagram ${ }^{\circledR} /$ Twitter $^{\circledR}$ ): Foi analisado também o compartilhamento nas redes sociais na web 2.0, avaliando se os dados de consulta livre, disponíveis na web, poderiam ser enviados diretamente para amigos e seguidores através de aplicações como o Facebook ${ }^{\circledR}$, Google $+^{\circledR}$, Instagram ${ }^{\circledR}$ e Twitter ${ }^{\circledR}$. Em alguns casos há opção de link com as redes sociais, mas como direcionamento da página do Facebook ${ }^{\circledR}$ ou Twitter $^{\circledR}$ do governo. Ou ainda encontrados casos de compartilhamento do link da página do portal da transparência, mas não dos dados consultados. Dos sites avaliados, apenas os portais de transparência de Minas Gerais, Paraíba e Alagoas apresentam a opção de compartilhamento direto da informação, através das redes sociais.

\section{QUADRO 7: Resumo das funcionalidades nos portais da transparência}

\begin{tabular}{|l|c|c|c|c|c|c|c|c|}
\hline Quantidade de Estados & \multicolumn{2}{|c|}{$\begin{array}{c}\text { Dados para con- } \\
\text { sulta livre }\end{array}$} & \multicolumn{2}{|c|}{$\begin{array}{c}\text { Email/ } \\
\text { Fale Conosco } \\
\text { Exclusivo }\end{array}$} & \multicolumn{3}{|c|}{ Chat } & \multicolumn{2}{c|}{$\begin{array}{c}\text { Compartilhamento } \\
\text { em Redes Sociais }\end{array}$} \\
\hline $\begin{array}{l}\text { Tem no portal da trans- } \\
\text { parência }\end{array}$ & 27 & $100 \%$ & 22 & $81 \%$ & 0 & $0 \%$ & 3 & $11 \%$ \\
\hline $\begin{array}{l}\text { Não tem no portal da } \\
\text { transparência }\end{array}$ & 0 & $0 \%$ & 5 & $19 \%$ & 27 & $100 \%$ & 24 & $89 \%$ \\
\hline Total & 27 & $100 \%$ & 27 & $100 \%$ & 27 & $100 \%$ & 27 & $100 \%$ \\
\hline
\end{tabular}

Fonte: Os Autores (20I6).

Conforme apresentado no Quadro 7, nenhum portal da transparência apresenta a possibilidade de comunicação através de chat e o compartilhamento através das redes sociais só foi observado em 3 portais. A opção de comunicação através do envio de emails ou preenchimento de formulário do fale conosco é a opção mais disponibilizada nos portais de transparência. $\mathrm{Na}$ avaliação dos 26 estados e do distrito federal, apenas 5 portais não apresentavam um email/fale conosco exclusivo para assuntos relacionados ao portal da transparência, mas apresentam um link de direcionamento para um email de ouvidoria ou outro órgão para receber a solicitação de informação. 


\section{Considerações Finais}

Temas relacionados às inovações tecnológicas e as mudanças nas interações entre os indivíduos e o governo oportunizadas ainda mais através da lei de acesso à informação (LAI) vêm abrindo direções para pesquisas com diversos focos de atuação. Em gestão e com foco na área de tecnologia da informação, é um tema que necessita de mais dados conceituais, fazendo assim com que novas pesquisas sejam necessárias para um melhor conhecimento da área integrando informação, comunicação e interatividade.

A partir dessa visão geral sobre a lei de acesso à informação, no setor público os desenvolvedores tem a oportunidade de avaliar novas possibilidades nos sistemas de comunicação e a disponibilização desses dados em outros meios e formatos, no setor privado o conhecimento dessas informações acrescenta na visão estratégica e de governança, bem como nas interações com o Governo. Alguns portais da transparência disponibilizam as informações, por exemplo, no formato do Microsoft Excel, o que facilita o uso desses dados para outras análises e geração de conhecimento.

A implementação da lei de acesso à informação ainda é um tema recente, sendo uma lei de 20 II ainda vem evoluindo no formato e conteúdo da disponibilização dos dados. De acordo com o relatório da CGU, em 20I4 apenas I9 estados estavam com a lei regulamentada (CGU,20I4) e atualmente observa-se que todos os estados e o Distrito Federal têm portais de transparência.

De toda forma, os cidadãos em geral passam a ter novas oportunidades de acesso e compartilhamento na web, no entanto, com o formato dos sites de transparência atuais, à questão da interatividade ainda tem muitas oportunidades de melhorias. De acordo com os resultados deste estudo, apenas em 3 casos foi possível compartilhar nas redes sociais os dados pesquisados, como uma planilha de despesas de determinado setor ou projetos realizados num período específico. O uso do chat para tirar dúvidas em tempo real não foi verificado em nenhum dos portais pesquisados, mas a maioria apresenta um formulário de fale conosco ou disponibiliza um email para contato com o usuário.

Observa-se que outros dados e notícias disponíveis em alguns portais de órgãos governamentais atualmente já incluem ferramentas de compartilhamento. Logo, os gestores públicos podem avaliar a viabilidade da inclusão de funcionalidades interativas nos portais da transparência que sejam de interesse da sociedade em geral para dar continuidade ao processo de mudança na cultura da informação no setor público. Como limitação deste estudo pode ser destacada a conceituação e delimitação das ferramentas interativas que foram avaliadas, bem como não foram testados se os portais que disponibilizam email e formulários de fale conosco respondem as solicitações enviadas. Em estudos futuros, outras ferramentas podem ser incluídas na análise, bem como um aprofundamento dos temas relacionados a interatividade e a gestão da informação no setor público. 


\section{REFERÊNCIAS}

BANNON, Lion J.; SCHMIDT, Kjeld. CSCW: Four Characters in Search of a Context. In: Studies in Computer Supported Cooperative Work. BOWERS, J.M.; BENFORD, S.D. (Org.). North Holland: Elsevier Science Publishers, I99I.

BRASIL. Lei $\mathbf{N}^{\mathbf{0}}$ I2.527 de I8 de novembro de 20II. Dispõe sobre os procedimentos a serem observados pela União, Estados, Distrito Federal e Municípios, com o fim de garantir o acesso a informações. Disponível em: http://www.planalto.gov.br/ccivil_03/_ato20II-20I4/20II/lei/li2527. htm. Acesso em: Io abr 2016.

BRASIL. Ministério da Transparência, Fiscalização e Controle. Escala Brasil Transparente. Disponível em: http://www.cgu.gov.br/assuntos/transparencia-publica. Acesso em: Io abr 2016.

BRITO, R. F.; PEREIRA, A. T. C. Um Estudo para Ambientes Colaborativos e suas Ferramentas. Anais... Congresso Nacional de Ambientes Hipermídia para Aprendizagem. Santa Catarina, 2004 .

CGU, Controladoria Geral da União. Cartilha de Acesso a Informação Pública: Uma introdução à Lei $\mathrm{n}^{\mathrm{o}}$ I2.527, de I8 de novembro de 20II. Disponível em: http://www.acessoainformacao.gov. br/central-de-conteudo/publicacoes/arquivos/cartilhaacessoainformacao.pdf. Acesso em: I6 abr 2016.

CGU, Controladoria Geral da União. Mapa de Transparência. Janeiro de 20I4. Disponível em: http:/www.cgu.gov.br/assuntos/transparencia-publica/brasil-transparente/mapa-transparencia/ mapa-da-transparencia-2. Acesso em: I6 abr 2016.

CRESWELL, J. W. Projeto de pesquisa: métodos qualitativo, quantitativo e misto. Porto Alegre: Artmed, 2010.

GIL, A. C. Como elaborar projetos de pesquisa. 4 ed. São Paulo: Atlas, 2009.

HAMPTON, D. R. Administração Contemporânea: teoria, prática e casos. 3 ed. São Paulo: Pearson Makron Books, 1992.

MARCONI, M. A.; LAKATOS, E. M. Metodologia científica. 5.ed. São Paulo: Atlas, 2007.

McMILLAN, Sally J. The Researchers and the Concept: Moving Beyond a Blind Examination of Interactivity. Journal of Interactive Advertising, v.5, n.2, p.I-4, 2005.

O'REILLY, Tim. What Is Web 2.o: Design Patterns and Business Models for the Next Generation of Software. 2005. Disponível em: http://oreilly.com/web2/archive/what-is-web-20. html. Acesso em: 2I mai 2016.

ORLIKOWSKI, Wanda J.; WOERNER, Stephanie L. Web 2.0: Experimenting With The Connected Web. CISR MIT Sloan Research Briefing, v. 9, n. 3, 2009. 
PARAMESWARAN, M.; WHINSTON, A. Social Computing: An Overview. Communications of the Association for Information Systems, v.I9, p.762-780, 2007.

RAFAELI, Sheizaf; SUDWEEKS, Fay. Networked Interactivity. Journal of Computer Mediated Communication, v.2, n.4, 1997.

ROBBINS, S. P. Comportamento Organizacional. Rio de Janeiro: LTC, 1999.

STONER, J. A. F. e FREEMAN, R. E. Administração. Rio de Janeiro: Prentice Hall, 1985.

STRAUSS, Anselm L.; CORBIN, Juliet M. Pesquisa qualitativa: técnicas e procedimentos para o desenvolvimento de teoria fundamentada. 2.ed. Porto Alegre: Artmed, 2008.

TAPIADOR, Antonio; FUMERO, Antonio; SALVACHÚA, Joaquín; AGUIRRE, Sandra. $A$ Web Collaboration Architecture. 2006. Disponível em: http://jungla.dit.upm.es/ saguirre/ publications/CollaborateCom2006_2.pdf. Acesso em: 2I out 2012. 